\title{
FEATURE-BASED DIGITAL WATERMARKING FOR REMOTE SENSING IMAGES
}

\author{
P.-H. Hsu ${ }^{\text {a, } *}$ and C.-C. Chen ${ }^{\mathrm{a}}$ \\ ${ }^{\text {a }}$ Dept. of Civil Engineering, National Taiwan University, No.1, Sec. 4, Roosevelt Rd., Taipei City 10617, Taiwan - \\ (hsuph, d98521018)@ntu.edu.tw
}

Commission III, ICWG III/VII

KEY WORDS: Watermarking, Feature, Extraction, Transformation, Quality, Robustness

\begin{abstract}
:
With the rapid development of information and communication technology, people can acquire and distribute many kinds of digital data more conveniently than before. The consequence is that the "copyright protection" which prevents digital data from been duplicated illegally should be paid much more attention. Digital watermarking is the process of embedding visible or invisible information into a digital signal which may be used to verify its authenticity or the identity of its owners. In the past, digital watermarking technology has been successfully applied to the "copyright protection" of multimedia data, however the researches and applications of applying digital watermarking to geo-information data are still very inadequate. In this study, a novel digital watermarking algorithm based on the scale-space feature points is applied to the remote sensing images, and the robustness of the embedded digital watermark and the impact on satellite image quality are evaluated and analysed. This kind of feature points are commonly invariant to Image rotation, scaling and translation, therefore they naturally fit into the requirement of geometrically robust image watermarking. The experiment results show almost all extracted watermarks have high values of normal correlation and can be recognized clearly after the processing of image compression, brightness adjustment and contrast adjustment. In addition, most of the extracted watermarks are identified after the geometric attacks. Furthermore, the unsupervised image classification is implemented on the watermarked images to evaluate the image quality reduction and the results show that classification accuracy is affected slightly after embedding watermarks into the satellite images.
\end{abstract}

\section{INTRODUCTION}

With the rapid development of information technology (IT) and communication technology (CT), people can acquire and distribute different kind of digital data more convenient than before. The consequence is that the "copyright protection" which prevent digital data from been duplicated illegally should be paid much more attention. Digital watermarking, which is a kind of information hiding technologies, is the process of embedding visible or invisible information into a digital signal which may be used to verify its authenticity or the identity of its owners. In the past, digital watermarking technology has been successfully applied to the "copyright protection" of various kinds of digital multimedia data, however the researches and applications of applying digital watermarking to geoinformation data are still very inadequate. In this study, the digital watermarking technology is applied to the remote sensing images, and the robustness of the embedded digital watermark and the impact on satellite image quality are evaluated and analysed.

A digital watermark is called robust if it could be detectable completely even when the watermarked data is subject to unintentional or intentional data modifications (or attacks). In addition to the robustness of watermark, the quality of original data after embedding the digital watermark also should be preserved. In order to satisfy these two basic requirements simultaneously, the digital watermarking algorithm should be developed according to the characteristics of the geospatial data and their applications. Hsu and Chen (2011) have proposed a novel algorithm that the digital watermark was embedded into the photogrammetric images, and the robustness of the embedded digital watermark and the impact on photogrammetric image quality are evaluated. In this study, this algorithm has been improved for the applications of satellite images. Firstly, the embedding and extracting algorithms of digital watermark should be robust to resist the geometric attacks such as image rotation, scaling, and translation (RST). Secondly, the digital watermark techniques should not result in the geometric deformation or radiometric distortion on the original image data. For satellite images, the geometric or radiometric distortion not only reduces the human's visual sensation but also affects the image processing results, such as the image matching, image classification and image measurements.

The robust image watermarking algorithm proposed in this study is based on the scale-space feature points to fulfill the two above-mentioned requirements. This kind of feature points are commonly invariant to rotation, scaling and translation, therefore they naturally fit into the requirement of geometrically robust image watermarking. For this reason, the scale-invariant feature transform (SIFT) is used to detect the scale-space feature points, than some non-overlapped circular regions surrounding the feature points are selected to embed the watermark. Furthermore, the watermark is adjusting the watermark intensity in accordance with the texture sensitive values of the wavelet packet transform of the circular regions to avoid the reduction of the satellite image quality.

In the experiments of this study, two data set from WorldView2 and Formosat- 2 satellites are used to test the performance of our proposed watermarking method. In the test of the watermark robustness, almost all extracted watermarks have high values of normal correlation and can be recognized clearly after the

\footnotetext{
* Corresponding author.
} 
processing of JPEG compression, brightness adjustment and contrast adjustment. However, it is not easy to identify the extracted watermark after the image smoothing. In addition, most of the extracted watermarks are identified after the geometric attacks such as the image rotation, scaling and translation (RST). Furthermore, the unsupervised image classification is implemented on the watermarked images to evaluate the image quality reduction. The results show that classification accuracy is affected slightly after embedding watermarks into the satellite images.

\section{DIGITAL WATERMARKING FOR REMOTELY SENSING IMAGES}

Digital watermarking for satellite imagery is the process of embedding visible or invisible information into the digital imagery which may be used to verify its authenticity or the identity of its owners. The embedded information can be the trademark, script, image chip, or any kind of digital information generated from the original images. A review of related research, and major study issues of digital watermarking for satellite images are described briefly as follows:

\subsection{Review of Related Researches}

Barni et al. (2002) have proposed a near-lossless watermarking algorithm using the discrete Fourier transform (DFT), discrete wavelet transform (DWT). The size of the watermark has been adjusted to test the intensity and robustness of watermark when an unsupervised classifier was performed on the satellite image. Ziegeler et al. (2003) have illustrated that the digital watermarking techniques developed for multimedia data cannot be directly applied to the satellite images due to the fact that the analytic integrity of the data, rather than perceptual quality, is of primary importance. Thus a DWT-based algorithm for the watermarking of remotely sensed images was proposed. The impact of watermarking by this algorithm on classification performance is evaluated. Kbaier and Belhadj (2006) also proposed a multispectral image watermarking algorithm based on DWT, the algorithm is robust to resist cropping and filtering attacks. Chen et al. (2010) have proposed a new watermarking algorithm based on block characteristics and discrete cosine transform, which reduces the impact of the watermarked satellite images. The proposed watermarking algorithm can resist image processing, such as added noise, cropping, filtering and compression, also can resist to geometric operate.

\subsection{Major Study Issues of Digital Watermarking}

The study of digital watermarking for satellite images should focus on the flowing issues:

1. The embedding watermark should not affect the content of the original satellite images in order to preserve the validity on the follow-up processing, such as the image matching, image classification and image measurements.

2. Geometric correction is an important process to reduce the satellite image distortions and establish the relationship between the image coordinate system and the geographic coordinate system. However, changing the pixel coordinates and image size also reduces the robustness of the embedded watermark. How to resist the attack of geometric correction will be an important issue of watermarking for satellite images.
3. In general, the watermarking algorithm based on the spatial domain is to directly modify the pixel values. On the other hand, the watermarking algorithm based on the frequency domain embeds the watermark into the frequency coefficients. Therefore, it would be difficult to resist the attacks of the image orthorectification which consists of the mosaicking and color balancing.

In this study, a novel watermarking algorithm based on the scale-space feature points is proposed to solve the above problems. The scale-space feature points are commonly invariant to image rotation, scaling and translating, therefore they naturally fit into the requirement of geometrically robust image watermarking.

\section{DIGITAL WATERMARKING ALGORITHM}

\subsection{Image Synchronization for Watermark Embedding and Extraction}

The watermark is always embedded into the image according to a certain sequence of pixels. The only way to extract the watermark exactly is following the same pixel sequence. If the watermark embedded image suffers from the changes of rotation or scaling attack, the pixel sequence consequently changes and causes the failure of watermark extraction. To resolve this problem, the pixel sequence should be synchronized when embedding and extracting the watermark.

In this study, we use the SIFT algorithm to find some keypoints distributed on the satellite image. These keypoints have the property of invariant on image scaling, rotation and brightness adjustment. Then the circular area which uses the keypoint as the center of the circle is selected to embed the watermark. When extracting the watermark, the same circular areas surrounding the same keypoints should be found. This can be done by using the dominant gradient orientation and characteristic scale of the keypoints.

\subsection{Selection of Watermark Embedding Area}

Using the SIFT algorithm, the keypoints and their characteristic scales $\sigma$ can be found out in the image. The circular areas surrounding the keypoints which can be used to resist the rotation attacks are constituted by the characteristic scale of the keypoint:

$$
\left(x-x_{0}\right)^{2}+\left(y-y_{0}\right)^{2}=(S \sigma)^{2}
$$

where $S$ is a parameter to adjust the radius of the circular area according to the characteristic scale of the keypoint. Figure 1(a) shows the circular areas selected by the characteristic scale and dominant gradient orientation of the keypoint (Tang and Hang, 2003). Since the circular areas of various keypoints are overlapped, 4 circular areas are selected to embed the watermark based on the demands on large characteristic scale and non-overlapping condition. In Figure 1(b), the direction of blue line shows the dominant gradient orientation of the keypoint. 


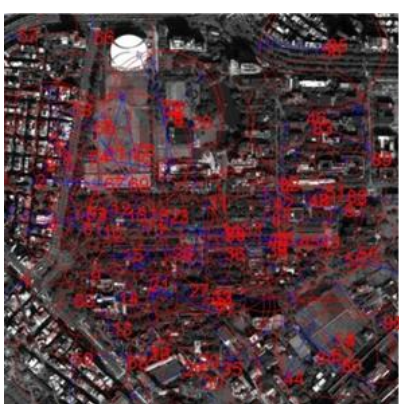

(a)

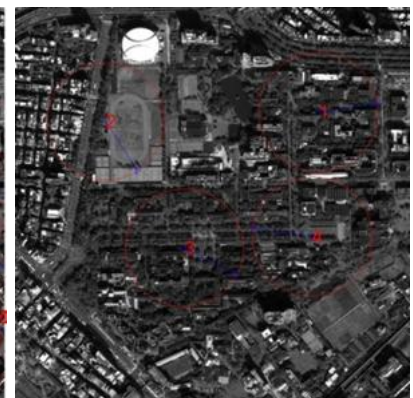

(b)
Figure 1. Circular areas selected by characteristic scale and dominant gradient orientation of keypoints (a) Overlapping circular areas (b) Non-overlapping circular areas

\subsection{Watermark embedding}

The steps of watermark embedding are described as follow:

1. Figure 2(a) shows the circular area selected by the feature point and figure 2(b) shows the result of the normalization according to the dominant gradient orientation. To avoid the damage on the feature points when embedding the watermark, a smaller circular area around the keypoint is removed which is shown in figure 2(c). Figure 2(d) shows the rectangular image after the circular image is reorganized.

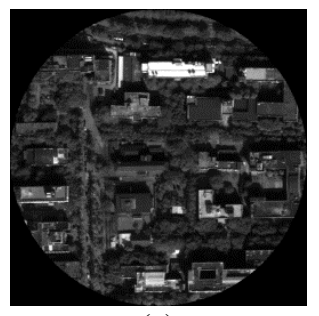

(a)

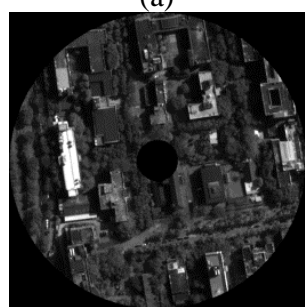

(c)

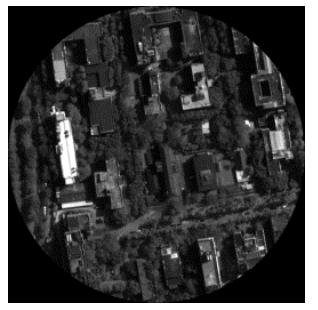

(b)

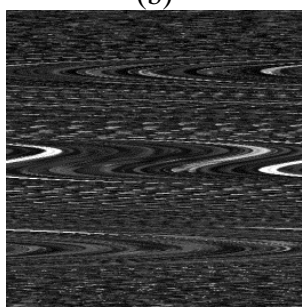

(d)
Figure 2. Selection, normalization, and rectangularity of the circular area by feature points.

2. Performing a three-layer wavelet packet transform (WPT) on the rectangular image, 64 sub-bands of wavelet coefficients can be calculated. In addition to the sub-band of low-frequency, the texture sensitivities of the other 63 subbands are calculated using equation (2) and (3).

$$
\begin{gathered}
T_{n}(i, j)=\sum_{P, Q=-1}^{1}|w(i+p, j+q)-B| \\
B=\sum_{P, Q=-1}^{1} w(i+p, j+q) / 9
\end{gathered}
$$

where $w(i, j)$ is the WPT coefficients on $(i, j)$, and B is the coefficients average adjacent to $(i, j)$.

3. Set a threshold $t_{f}$, and using equation (4) to calculate the number of texture sensitivity $N_{n}(T)$ within the $n^{\text {th }}$ sub-band. Then the sub-band with maximum number of texture sensitivity will be selected to embed watermark.

$$
N_{n}(T)=\operatorname{number}\left\{\mathrm{T}_{\mathrm{n}}(\mathrm{i}, \mathrm{j})>t_{f}\right\}
$$

4. According to the selected sub-band, using formula (5) to calculate the energy E, $m \times n$ is the size of the sub-band.

$$
\mathrm{E}=\sum_{\mathrm{i}=1}^{\mathrm{m}} \sum_{\mathrm{j}=1}^{\mathrm{n}} \mathrm{w}^{2}(i, j)
$$

5. Calculate the embedded intensity using equation (6), where $d_{f}$ is an empiric value for intensity adjustment.

$$
a_{n}=\frac{\mathrm{N}_{\mathrm{n}}(\mathrm{T})}{\mathrm{E}} d_{f}=\frac{\text { number }\left\{\mathrm{T}_{\mathrm{n}}(\mathrm{i}, \mathrm{j})>t_{f}\right\}}{\sum_{\mathrm{i}=1}^{\mathrm{m}} \sum_{\mathrm{j}=1}^{\mathrm{n}} \mathrm{w}^{2}(i, j)} d_{f}
$$

6. Divide the selected sub-band into $2 \times 2$ blocks, and using equation (7) and (8) to modify the upper left coefficient of each block to embed watermark.

$$
\begin{aligned}
& \text { if the value of embedding watermark is } 1 \text {, } \\
& w^{\prime}(i, j)=\max \{w(i, j) i=1,2 ; j=1,2\}+a_{n} \\
& \text { if the value of embedding watermark is } 0, \\
& w^{\prime}(i, j)=\min \{w(i, j) i=1,2 ; j=1,2\}-a_{n}
\end{aligned}
$$

7. Perform the inverse wavelet packet transform on the coefficients after embedding the watermark, the circular image containing watermark is obtained. Then rotate the circular image inversely according to the dominant gradient orientation. Finally, fill the circular image back to the original satellite image then an image with watermark embedded around the local keypoints is obtained.

\subsection{Watermark Extraction}

The steps of watermark extraction are illustrated as follow:

1. Make use of SIFT algorithm to get the feature points of satellite image containing watermark, gain the circular image based on the characteristic scale $\sigma$ and dominant gradient orientation and then perform the image normalization, and remove annular image centered on the feature point, take it as the areas for watermark extraction and reorganize the annular image as the rectangular image.

2. Perform a three-layer wavelet packet transform on the rectangular image and obtain 64 sub-bands, select the subbands with maximum number of texture sensitivity.

3. Divide the selected sub-band into $2 \times 2$ block, and using the formula (9) to calculate the value of $\mathrm{T}$.

$$
\begin{aligned}
\mathrm{T}= & \{\max \{w(i, j) i=1,2 ; j=1,2\} \\
& +\min \{w(i, j) i=1,2 ; j=1,2\}\} / 2
\end{aligned}
$$


Using the upper left coefficients of each block to extract the watermark information, when $w^{\prime}(i, j) \geq T$, the value of embedding watermark is 1 , when $w^{\prime}(i, j) \leq T$, the value of embedding watermark is 0 .

\subsection{Assessment Criteria of Watermark Algorithm}

\subsubsection{Quality Indicators of Watermarked Images}

In order to evaluate the impact of the watermark on the original satellite images, the mean square error (MSE) and the peak signal-to-noise ratio (PSNR) between the image embedded with watermark and the original image. If the mean square error is small or the peak signal-to-noise ratio is large, then we can determine that the similarity of the two images is high.

$$
\begin{aligned}
& \operatorname{MSE}=\left(\frac{1}{m \times n}\right) \sum_{i=0}^{m-1} \sum_{j=0}^{n-1}\left(I_{i j}-I_{i j}^{\prime}\right)^{2} \\
& \text { PSNR }=10 \log _{10} \frac{255^{2}}{M S E} d B
\end{aligned}
$$

where $I_{i j}$ and $I_{i j}^{\prime}$ represents respectively the pixel values of the original image and the image embedded with watermark.

\subsubsection{Robustness Verification of Watermark}

In this study, the $\mathrm{NC}$ value in equation (12) is calculated to verify the robustness of the watermark. If the $\mathrm{NC}$ value (within $0 \sim 1)$ is large, then the extracted watermark has a low degree of distortion or high livability, which can be clearly identified by the human eyes.

$$
\mathrm{NC}=\frac{\sum_{0}^{\mathrm{m}-1} \sum_{0}^{\mathrm{m}-1} \mathrm{~W}(\mathrm{i}, \mathrm{j}) \oplus \mathrm{W}^{*}(\mathrm{i}, \mathrm{j})}{\mathrm{m} \times \mathrm{m}}
$$

where $\mathrm{W}$ and $\mathrm{W}^{*}$ are respectively the original watermark and the extracted watermark suffered from various attacks, $\oplus$ is exclusive-or (XOR) operator.

\section{EXPERIMENTS}

The test image in this experiment is a subset of WorldView-2 with the size of $2000 \times 2000$, which is shown in figure 3 (a). The selected watermark content is the Chinese characters of "National Taiwan University" coordinated with individual document No., which is specified as "1001227", so as to act as the declaration of the ownership and distinguish different users. The contents of watermark are shown in figure 4(a), the size of watermark image is $40 \times 40$

\subsection{Quality and Robustness of Watermarked Images}

Figure 3(a) shows the original image and figure 3(b) shows the WorldView-2 image embedded with watermark. The PSNR value of image embedded with watermark is 47.59 , and the average PSNR of the four watermarked areas is 40.7, which mean the distortion of image embedded with watermark is not evident. The size of the embedded watermark is $31 \times 31$. If the image embedded with watermark doesn't suffer from any attack. Figure 4(b) shows the extracted watermark with the NC value of 0.998 .

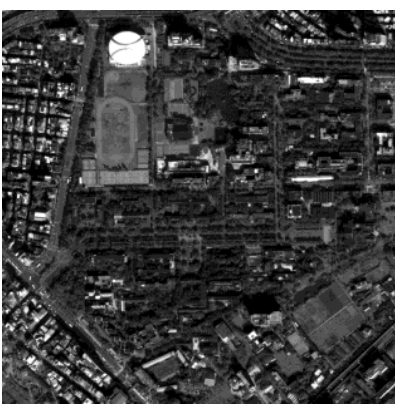

(a)

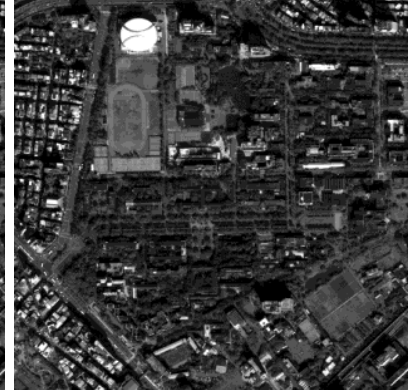

(b)
Figure 3. (a) Contents of original image and (b) Watermarked image

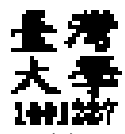

(a)

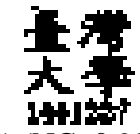

(b) $(\mathrm{NC}=0.998)$
Figure 4. (a) Original watermark and (b) Extracted watermark

Figure 5 shows the results of robust verification of watermark after the JPEG compression, smoothing, brightness adjustment, regulation, contrast, resizing, translation, rotation and cutting. It can be found from the extracted watermark in figure 5 that, the watermark algorithm proposed in this study can resist general JPEG compression, brightness adjustment, regulation and contrast, as well as various geometric attacks such as image resizing, translation, rotation and cutting, the extracted watermark can achieve very good recognizable.

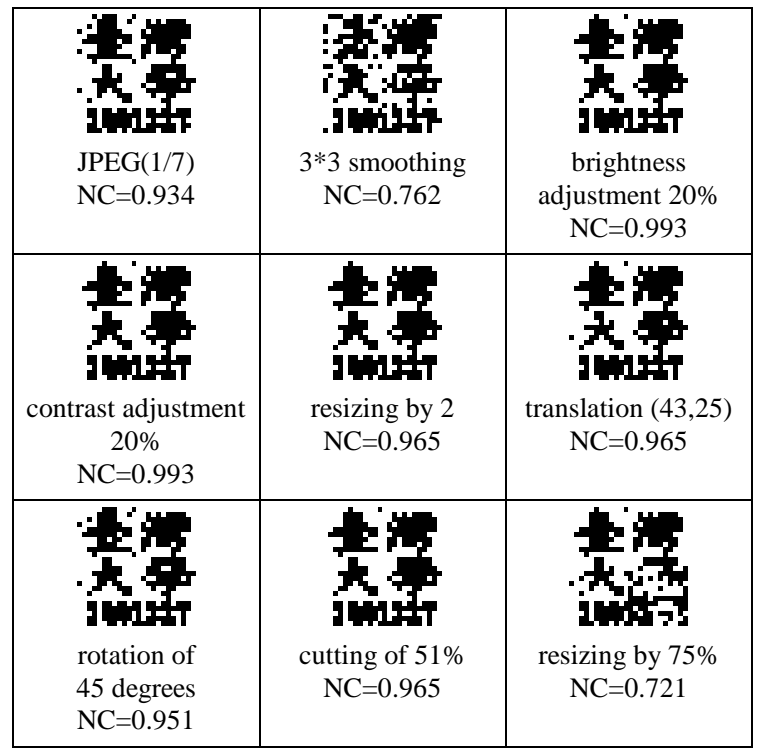

Figure 5. Resistance ability of No. 4 area after various image processing.

In order to verify the robustness of the watermark after the geometric correction, the RST and polynomial transformation are performed respectively on the test image using 6 ground control points. The results are shown in figure (6) and (7). Figure 7 (a) is the watermark extracted from the region of No. 2 using RST transformation. The NC value of the extracted watermark is 0.899 and can be identify with ease. However, the extracted watermark after polynomial transformation is unidentifiable and has a small NC value of 0.307 . 


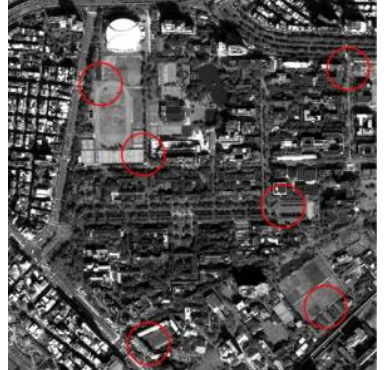

(a)

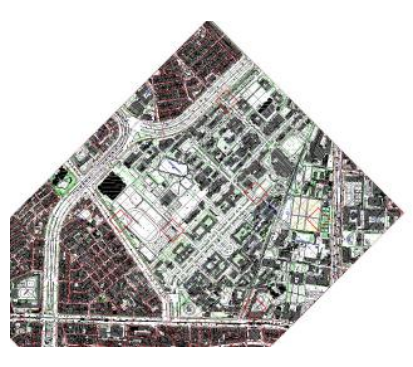

(b)
Figure 6. Geometric correction of watermarked image : (a) Selected points on watermarked image, (b) Selected points on digital map

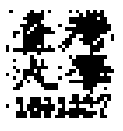

(a)

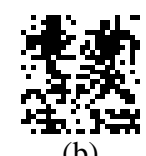

(b)
Figure 7. (a) $\mathrm{NC}(=0.899)$ of $\mathrm{RST}(\mathrm{b}) \mathrm{NC}(=0.307)$ of polynomial.

\subsection{Analysis of Texture Sensitivity and Watermark Intensity}

Table 1 shows relationship between the number of texture sensitivity and PSNR and NC. One may notice that No. 2 has the maximum number of texture sensitivity. When embedding the watermark with the fixed intensity previously designed, the deformation of No. 2 is the largest (with smallest value of PSNR) comparing to the others, however the NC values for different attacks are the highest when extracting the watermark. On the other hand, No. 3 has the smallest number of texture sensitivity, the deformation is smaller when embedding the watermark with the same intensity previously designed. However, the small $\mathrm{NC}$ values also indicate the reduced robustness of watermark. According to this phenomenon, an adaptive satellite image watermarking algorithm based on the number of texture sensitivity has been proposed by adjusting the watermark intensity. The adjusted value of watermark embedding intensity is showing in Table 2 , the deformation and robustness results of embedded watermark are shown in Table 3. The results show that satellite image distortion with a lower amount after embedding watermark, using the texture sensitive value to adjust intensity can get more robust and lower deformation results.

\begin{tabular}{|l|cccc|}
\hline No. of Circular Area & 1 & 2 & 3 & 4 \\
\hline Number of Texture Sensitivity & 148 & 154 & 33 & 74 \\
PSNR & 39.70 & 39.03 & 43.36 & 41.51 \\
NC of No Attack & 0.994 & 0.998 & 0.975 & 0.998 \\
NC of 3*3 Smoothing & 0.767 & 0.821 & 0.621 & 0.762 \\
NC of RST Geometric correction & 0.798 & 0.899 & 0.6277 & 0.751 \\
\hline
\end{tabular}

Table 1. Deformation and robustness of various texture sensitive values after embedded watermark

\begin{tabular}{|c|ccc|}
\hline Number of Texture Sensitivity & $1-50$ & $51-100$ & $>100$ \\
\hline Watermark Embedding Intensity & 2 & 0.8 & 0.5 \\
\hline
\end{tabular}

Table 2. Adjust the watermark embedding intensity according to the texture sensitive values

\begin{tabular}{|c|cccc|}
\hline No. of Circular Area & 1 & 2 & 3 & 4 \\
\hline Number of Texture Sensitivity & 148 & 154 & 33 & 74 \\
PSNR & 41.15 & 40.58 & 41.75 & 42.00 \\
NC of No Attack & 0.991 & 0.992 & 0.994 & 0.998 \\
NC of 3*3 Smoothing & 0.812 & 0.852 & 0.874 & 0.854 \\
NC of RST Geometric correction & 0.821 & 0.881 & 0.835 & 0.802 \\
\hline
\end{tabular}

Table 3. Adjust the watermark embedding intensity according to the texture sensitive values of WorldView-2 image

\subsection{Influence on Image Classification}

Image classification is one of the most important applications of remote sensing. Because most of the image classification methods are based on the variation of the pixel values in each band, the change of the pixel value due to the watermark embedding may reduce the classification accuracy. In order to evaluate the influence of watermark on image classification, the $\mathrm{k}$-means method which is a kind of unsupervised classifiers is performing on the original image and the watermarked image respectively. The classification results are shown in figure 7 and table 4. One may find the greatest difference of percentage is only $0.04 \%$, which means that embedding watermark into satellite images has little effects on subsequent classification.

\begin{tabular}{|c|cccc|}
\hline No. of Circular Area & 1 & 2 & 3 & 4 \\
\hline Number of Differences & 1580 & -1779 & 342 & 1151 \\
Original Image (\%) & 19.59 & 12.50 & 13.08 & 13.81 \\
Watermark Image (\%) & 19.55 & 12.55 & 13.07 & 13.78 \\
Difference of Percentage (\%) & 0.04 & -0.04 & 0.01 & 0.03 \\
Number & 5 & 6 & 7 & 8 \\
Number of Differences & -1544 & 982 & -1059 & 327 \\
Original Image (\%) & 11.43 & 10.02 & 5.88 & 13.70 \\
Watermark Image (\%) & 11.47 & 9.99 & 5.90 & 13.69 \\
Difference of Percentage (\%) & -0.04 & 0.02 & -0.03 & 0.01 \\
\hline
\end{tabular}

Table 4. Effect of watermarking on unsupervised image classification using WorldView-2 Image

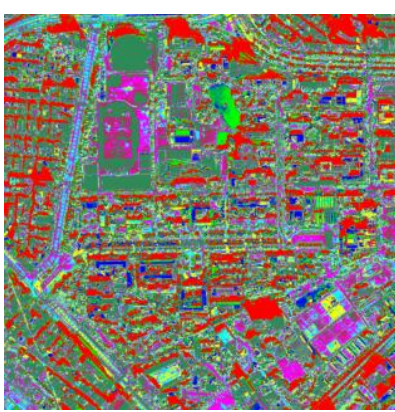

(a)

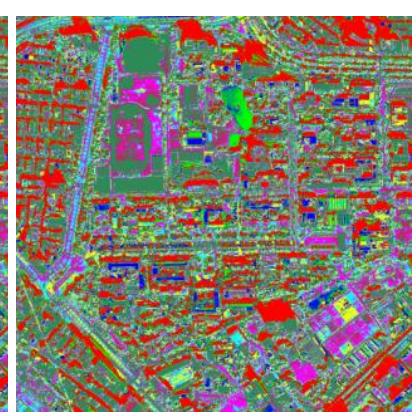

(b)
Figure 7. Classification results of the original image and watermarked image 


\subsection{Comparative Analysis with Formosat-2 Image}

The purpose of this experiment is to test the performance of the proposed watermarking algorithm on different kind of satellite images with different spatial resolution. The test image in this experiment is captured by Formasat-2, the image size is $2000 x$ 2000 with ground resolution of $2 \mathrm{~m}$. The watermark content and parameters are the same with the previous testing of WorldView-2 satellite images. Figure 7 shows the circular areas of the Formosat-2 image selected from the characteristic scale of the feature points, and only four circular areas are used to embed watermark. The relationship between number of texture sensitivity, deformation, intensity, and robustness of the watermark are listed in Table 5. In table 5, the original threshold for the WorldView-2 images does not suit the Formosat-2 images, resulting in each block of texture sensitive value is too large, although the intensity and robustness after the attack has performed extremely well, but the intensity of the embedded watermark is too large, image distortion after the watermarked is very bigger. According to this characteristic, we adjusted the critical value of the watermarking algorithm, and adjust the embedded watermark intensity according to Table 6 . The deformation and robustness of watermarked as shown in Table 7, although the intensity and robustness of the performance is slightly lower, but the deformation amount of improvement is significant.

\begin{tabular}{|l|cccc|}
\hline No. of Circular Area & 1 & 2 & 3 & 4 \\
\hline Number of Texture Sensitivity & 561 & 375 & 232 & 300 \\
PSNR & 35.84 & 36.72 & 38.19 & 37.23 \\
NC of No Attack & 1 & 0.998 & 1 & 0.998 \\
NC of 3*3 Smoothing & 0.785 & 0.757 & 0.786 & 0.853 \\
\hline
\end{tabular}

Table 5. Deformation and robustness of various texture sensitive values of the Formosat-2 image after embedded watermark

\begin{tabular}{|c|ccc|}
\hline Number of Texture Sensitivity & $1-50$ & $51-100$ & $>100$ \\
\hline Watermark Embedding Strength & 1.5 & 1 & 0.3 \\
\hline
\end{tabular}

Table 6. Adjust the watermark embedding intensity according to the texture sensitive values of the Formosat-2 image

\begin{tabular}{|l|rrrr|}
\hline No. of Circular Area & 1 & 2 & 3 & 4 \\
\hline Number of Texture Sensitivity & 277 & 150 & 70 & 116 \\
\hline PSNR & 38.47 & 39.38 & 40.33 & 40.18 \\
\hline NC of No Attack & 0.988 & 0.968 & 0.992 & 0.941 \\
\hline NC of 3*3 Smoothing & 0.665 & 0.662 & 0.739 & 0.675 \\
\hline
\end{tabular}

Table 7. Deformation, robustness of adjust the watermark embedding intensity according to the texture sensitive values of the Formosat-2 image

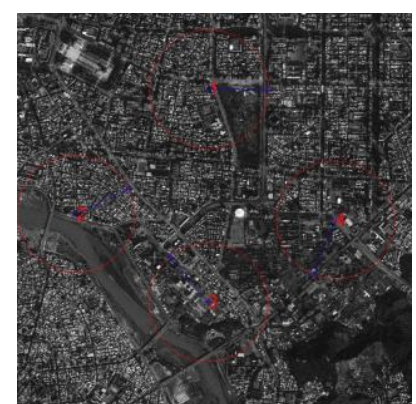

Figure 8. Circular areas of the Formosat-2 image selected by characteristic scale and dominant gradient orientation of

Keypoints

\section{CONCLUSION AND FUTURE GOALS}

In this study, a novel watermarking algorithm based on the scale-space feature points is proposed for remotely sensing images. The proposed watermarking algorithm has been tested on WorldView-2 and Formosat-2 image set. The results show that the proposed method can resist most of the attacks caused by the follow-up image processing, such as the image compression, brightness and contrast adjustment. In addition, thet embedding watermark into remotely sensing images only has slight influence on image classification accuracy.

Furthermore, the experiment results also show that the watermark robustness can be preserved when simple geometric processing is performed on the satellite images with watermarking embedded. However, the extracted watermark after polynomial transformation is unidentifiable and has a small NC value. This indicates that this watermarking algorithm still not have the ability to resist the polynomial geometric correction. In the future, the watermark algorithm will be improved to make it more suitable for the copyright protection of remotely sensing images

\section{REFERENCES}

Barni, M., F. Bartolini, V. Cappellini, E. Magli, and G. Olmo, 2002. Near-lossless digital watermarking for copyright protection of remote sensing images, Proceedings of the IGARSS 2002, Toronto, Canada, Vol. 3, pp. 1447-1449.

Chen, Q., P. P. Xie, W. J. Ma, W. Y. Wei, and L. H. Ai, 2010. A digital watermarking algorithm based on characters of the remote-sensing imagery, International Conference on MASS 2010 , Wuhan, China, pp. 1-4.

Hsu, P.-H. and C.-C. Chen, 2011. Study and analysis of digital watermarking for photogrammetric images", Proceedings of 32th Asian Conference on Remote Sensing, Taipei, Taiwan, TS4-9.

Kbaier, I., and Z. Belhadj, 2006. A novel content preserving watermarking scheme for multipectral images, Proceedings of the ICTTA 2006 , Damascus, pp.322-327.

Tang, C. W., and H. M. Hang, 2003. A feature-based robust digital image watermarking scheme, IEEE Transactions on Signal Processing, 51(4), pp. 950 - 959.

Ziegeler, S. B., H. Tamhankar, J. E. Fowler, and L. M. Bruce, 2003. Wavelet-based watermarking of remotely sensed imagery tailored to classification performance, Proceedings of the IEEE Workshop on Advances in Techniques for Analysis of Remotely Sensed Data, Washington D.C., pp. 564-579. 